Wes McGee Monica Ponce de Leon Editors

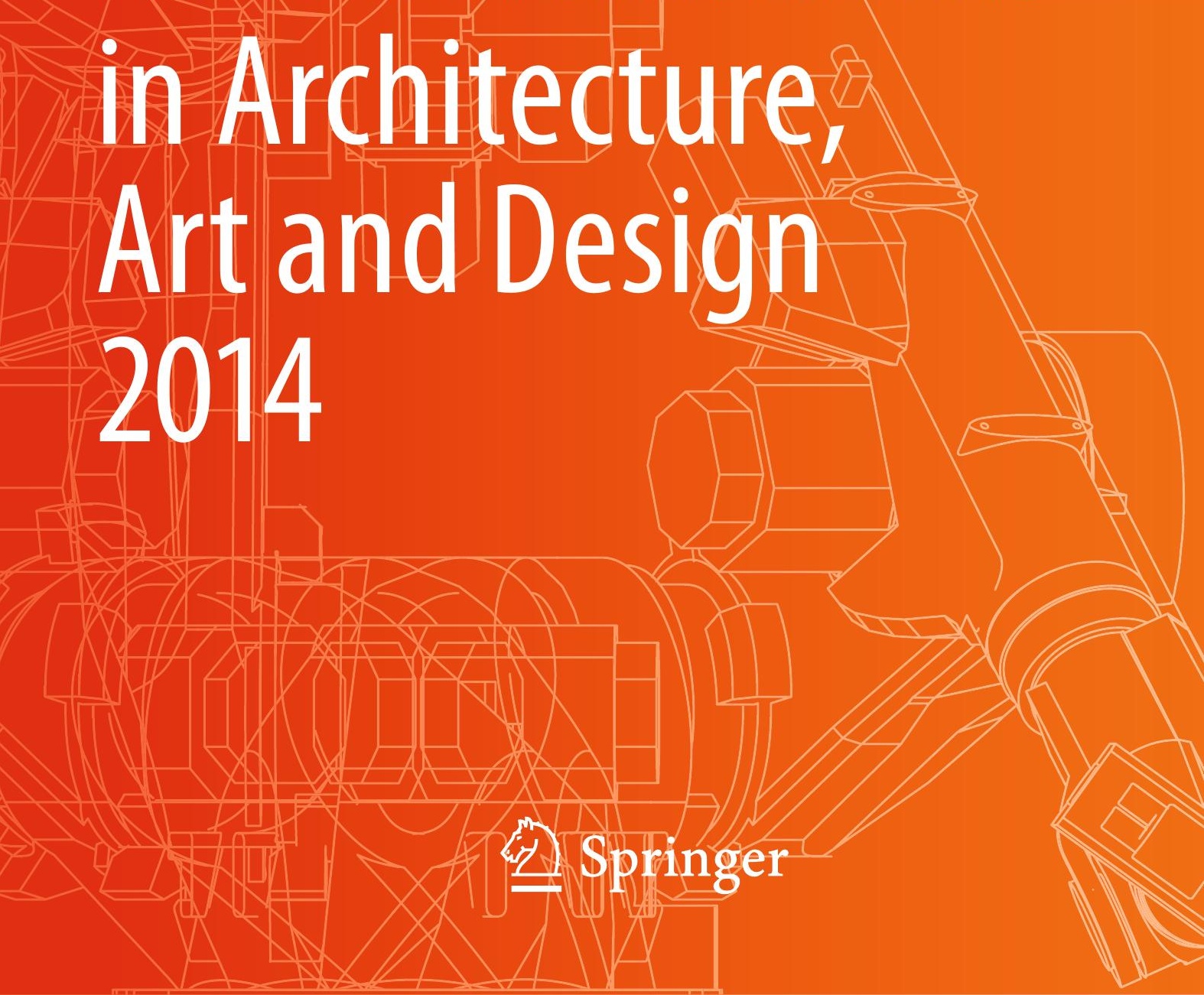
Robotic Fabrication in Architecture Art a
2014 


\title{
Design and Fabrication of Robot-Manufactured Joints for a Curved-Folded Thin-Shell Structure Made from CLT
}

\author{
Christopher Robeller, Seyed Sina Nabaei and Yves Weinand
}

\begin{abstract}
The prototype presented in this chapter utilizes the technique of curved folding for the design of a thin-shell structure built from curved cross-laminated timber panels (CLT). The curved-folded geometry allows for a span of $13.5 \mathrm{~m}$, at a shell thickness of only $77 \mathrm{~mm}$. The construction requires curved line CLT joints, which are difficult to address with state-of-the-art jointing techniques for CLT. Inspired by traditional woodworking joinery, we have designed connections for the integrated attachment of curved CLT panels, utilizing digital geometry processing tools to combine the advantages of traditional joinery techniques with those of modern automation technology.
\end{abstract}

Keywords Curved folded plate structures - Robot fabrication - Cross-laminated timber • Mono-material

\section{Motivation}

Recent studies about folded plate structures such as the Chapel St. Loup (Buri and Weinand 2010) and the "ICD/ITKE Research Pavilion 2011" (Magna et al. 2013), have demonstrated architectural and structural applications of folded-plate structures built from plywood and cross-laminated timber panels (CLT). Architectural

C. Robeller $(\square) \cdot$ S. S. Nabaei $\cdot$ Y. Weinand

EPFL Laboratory for Timber Construction IBOIS, Lausanne, Switzerland

e-mail: christopher.robeller@epfl.ch

S. S. Nabaei

e-mail: sina.nabaei@epfl.ch

Y. Weinand

e-mail: yves.weinand@epfl.ch 


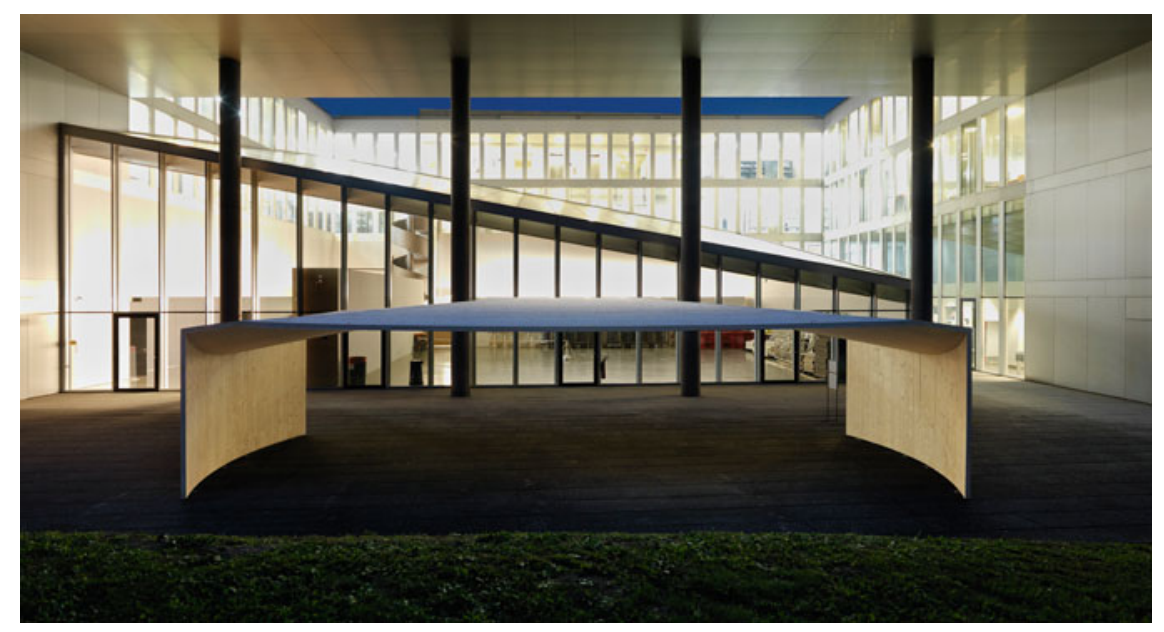

Fig. 1 Full-scale, mono-material prototype structure, spanning $13.5 \mathrm{~m}$ at a shell thickness of $77 \mathrm{~mm}$, exhibited at the academy of architecture in Mendrisio, Switzerland

applications of curved-folded geometry have previously been studied for benefits such as the panelization of complex shapes (Kilian et al. 2008).

The curved-folded thin-shell structure presented in the present chapter builds up on this research and demonstrates a structural application of curved CLT, a relatively new but increasingly popular product offered by European CLT manufacturers. Our prototype (Fig. 1) utilizes the curved shape of the panels to increase the inertia of the structure in its main load-bearing direction, acting very much the same way as a bi-cantilever frame. Our interpretation of the traditional simple frame is meanwhile innovative using curved thin-shell panels instead of beams, which are transmitting structural efforts in a curved line of action through robot fabricated wood working joints.

Moreover, connecting two reversely curved panels through a curved fold would propagate the curvature from one into another when one of them is bent. This interaction helps resisting lateral forces which tend to flatten their curvature. These lateral forces are transformed into out of plane forces due to the shell behavior of panels, at the interface and transmitted through the curved panels until the clamped edge.

Alternatively, such deformations are supported in practice by tensile steel cables along the chord of the curved CLT elements, as demonstrated in the recent wide-span roof construction of a factory building in Ainet, Austria (http://www. holzbau-unterrainer.at/Hallenbau). Instead of tensile cables, our prototype supports these forces by taking advantage of the curved folding technique.

Unlike origami paper folded forms, wood panels cannot actually be "folded". Instead, the performance of such structures depends on linear edge-to-edge connections between the panels, making these joints a key component in the design of wooden folded-plate structures. 
(a)

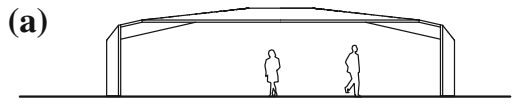

(c)



(b)

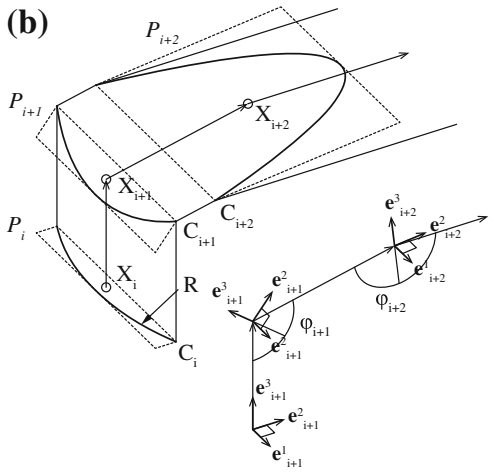

Fig. 2 a Full-scale prototype side view schematic. b Curved-folding technique. c Curved-folded thin-shell geometry. d Unfolded CLT parts/fabrication layout

\section{Project Geometry}

As illustrated in Fig. 2b, the curved-folded geometry is generated through a circular $\operatorname{arc} \mathrm{C}_{\mathrm{i}}($ Radius $\mathrm{R}=5.9 \mathrm{~m})$ and its reflection about a set of planes $\mathrm{P}_{\mathrm{i}}$. These reflection planes are located at the nodes $\mathrm{X}_{\mathrm{i}}$ of a polyline, which lies on a vertical plane normal to the chord of $\mathrm{C}_{\mathrm{i}}$. Each Reflection Plane $\mathrm{P}_{\mathrm{i}}$ is normal to the bisector of the two neighbor segments of the polyline. The fold angles $\varphi_{\mathrm{i}}$ are determined by the interior angles between the polyline segments. We used this polyline as a variable control polygon in a parametric design model.

The unfoldable geometry (Fig. 2d) allows for an efficient fabrication without offcuts. Depending on the length of the panel manufacturer's production line, all parts can be produced from a single, $17 \mathrm{~m}$ long curved CLT panel.

\section{Limitations of State-of-the-Art Connectors}

Figure $2 \mathrm{~d}$ illustrates the four connections $\mathrm{W}_{1}-\mathrm{CV}_{1}, \mathrm{CV}_{1}-\mathrm{CX}, \mathrm{CX}-\mathrm{CV}_{2}$ and $\mathrm{CV}_{2}-$ $\mathrm{W}_{2}$ that are required for the assembly of the curved-folded CLT thin-shell. The curved panels must be jointed at precise, non-orthogonal angles, which are difficult to address with state of the art CLT jointing techniques: Glued-in metal connectors, which are common in glulam and LVL constructions, cannot be applied to CLT because of its internal stress cuts, into which the adhesive could discharge.

Alternatively, external metal plate connectors could be applied visibly on top of the panels with wood screws. A large amount of such connectors would be required in order to achieve a sufficiently rigid and stiff joint. In general, the before mentioned connectors reduce the stiffness of a folded-plate, while modern wood adhesives, such as 1K-PUR, allow for a lossless, fully stiff joint. 
However, the gluing procedure requires a fast and precise alignment and clamping of the parts, within the open time of the adhesive. For joints on orthogonal edges and flat panels, standard tools can be used for the alignment and clamping. However, non-orthogonal edges and bent or curved panels will require custom made guides or supports.

\section{Joint Types and Algorithms}

Inspiration for an integrated solution for alignment and attachment can be found in traditional wood joinery techniques such as dovetail joints, on which the prismatic edge geometry results in a single-degree-of-freedom (SDOF) joint, which predetermines the alignment and orientation of the two connected parts. Due to their involving manual fabrication, these traditional joints have lost importance during the industrialization. Later on, early mass-production technology was incapable of fabricating such connections.

Modern robot- and CNC fabrication technology however allows for the reconsideration of these techniques. An application on plywood panels has been demonstrated recently (Simek and Sebera 2010) as well as a spotfacing technique for the robot fabrication of finger joints on plywood (Magna et al. 2013).

On our curved-folded prototype structure, we have to address two different types of folds. For the joints $\mathrm{W}_{1}-\mathrm{CV}_{1}$ and $\mathrm{CV}_{2}-\mathrm{W}_{2}$ each with a fold angle $\varphi$ of 102. $7^{\circ}$, we have chosen a SDOF dovetail-type joint geometry (Fig. 3).

The geometry is generated by an algorithm, which divides the joint edge into an odd number of segments, returning a set of points $X_{i}$. A local frame $\left\{\mathbf{u}_{i}^{1}, \mathbf{u}_{i}^{2}, \mathbf{u}_{i}^{3}\right\}$ is added to these points, where and $\mathbf{u}_{i}^{2}$ is parallel to the $\mathbf{e}^{2}$ and $\mathbf{u}_{\mathrm{i}}^{1}$ is the direction of insertion for the joint. These frames are rotated about $\mathbf{u}_{\mathrm{i}}^{1}$ at an alternating angle $\pm \theta$. For each frame, a plane $P_{\mathrm{Xi}}$, normal to $\mathbf{u}_{\mathrm{i}}^{3}$ is intersected with the curves $\mathrm{L}_{1}, \mathrm{~L}_{2}, \mathrm{~L}_{3}, \mathrm{~L}_{4}$, which gives us the curve and line segments for the dovetail joint geometry (Fig. 3b).

For the fabrication of these joints, we have chosen a side-cutting technique, using a shank type cutter (Koch 1964). The cutting requires simultaneous 5x machining, for which we provide a set of tool center points $\mathrm{TCP}_{\mathrm{i}}$, each with a direction $\mathbf{m}_{\text {orientation }}$ (Fig. 4, right side). We have obtained these points and vectors through an algorithmic evaluation of a set of parametric surfaces $S_{i}$.

The algorithm detects inside corners automatically and adds notches (Fig. 4, right side), which allow for the insertion of the joint's counterpart and reduce the notch stress (Simek and Sebera 2010; EN 1995-1-1 2004). The transition of the cutter between joint faces with different orientations is solved through the bisectorplanes $\mathrm{P}_{\mathrm{i}}$.

As one of its main advantages, the side-cutting technique allows for the use of simple supports under the workpiece. It is not necessary for the edge to cantilever. The main limitation for this method is set by the geometry of the cutter and the tool holder: The inclination $\beta$ of the tool must be limited to avoid a collision 


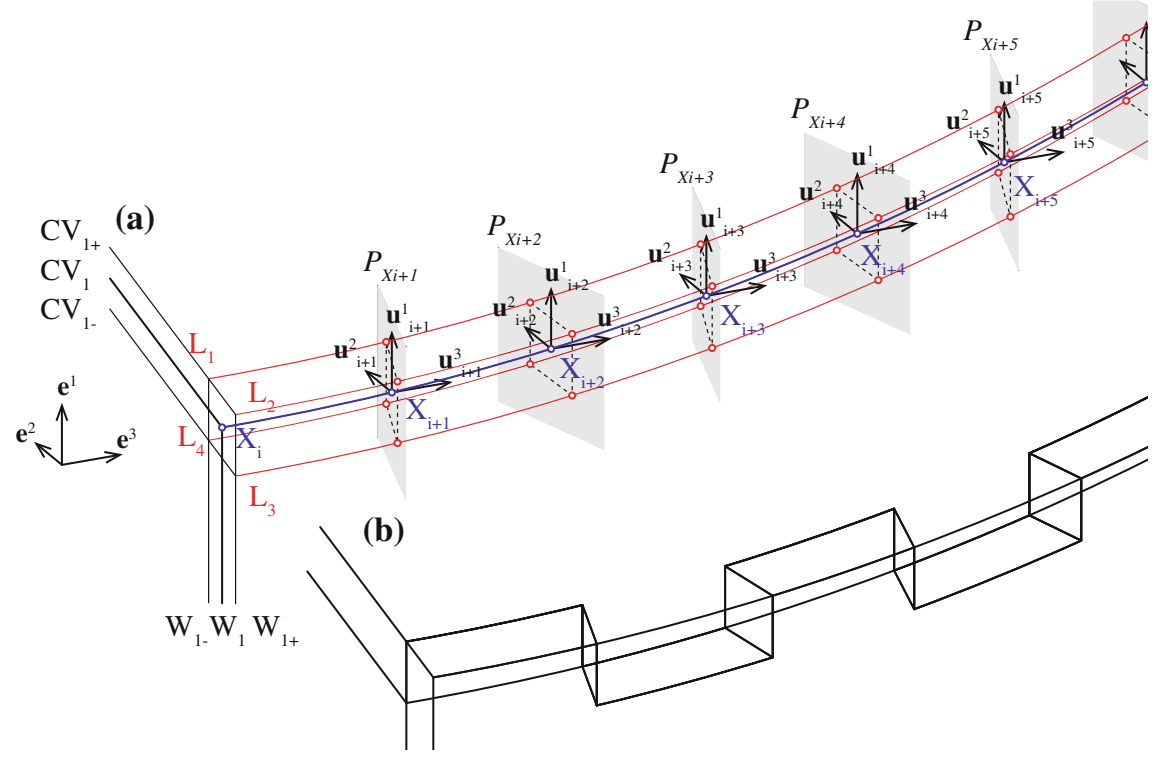

Fig. 3 Generation of the dovetail-type joint SDOF geometry through intersection of a set of planes $\mathrm{P}_{\mathrm{Xi}}$ with the four curves $\mathrm{L}_{1}, \mathrm{~L}_{2}, \mathrm{~L}_{3}$ and $\mathrm{L}_{4}$. a $\mathrm{CV} 1$ is the part, the + and - show the intersection layers and $\mathrm{W} 1$ is the part, the + and - show the intersection layers $\mathbf{b}$ it is the same parts once jointed. Part CV1 above and part W1 below

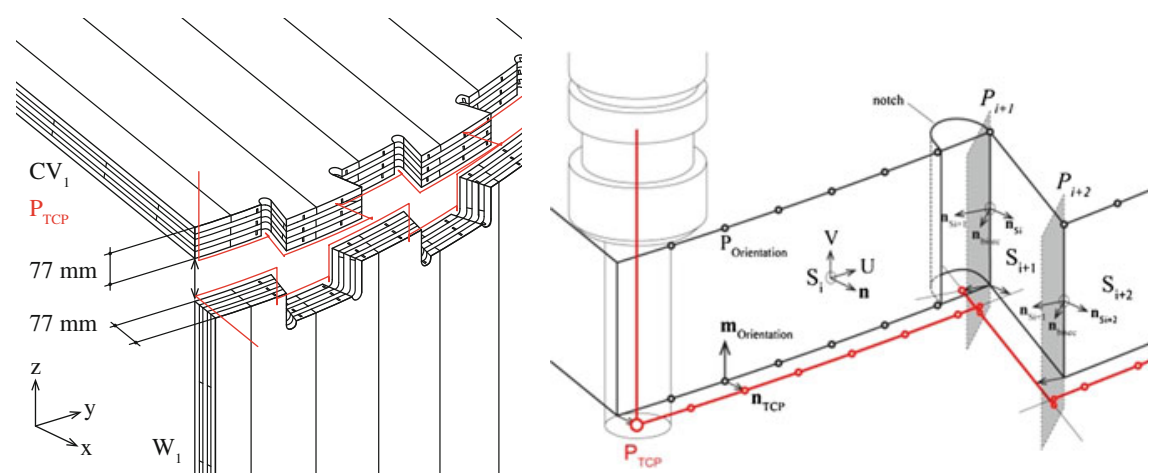

Fig. 4 Dovetail-type connections $\mathrm{W}_{1}-\mathrm{CV}_{1}$ and $\mathrm{CV}_{2}-\mathrm{W}_{2}$ on the full-scale prototype. Left schematic drawing showing the CLT panels and joints including layers and stress cuts, Right principle of machine code generation on a curved joint, using parametric surface evaluation

between the cutter and the wood panel. In consequence, this method can be applied to fold angles $\varphi$ within the range of $90^{\circ} \pm \beta$. With $\beta=40^{\circ}$ in our case, we are limited to folds up to $\varphi=130^{\circ}$. For the Connections $\mathrm{CV}_{1}-\mathrm{CX}$, and $\mathrm{CX}-\mathrm{CV}_{2}$, each with fold angle $167.2^{\circ}$, we have generated the joints with the method described in Fig. 3 , but without a rotation about $\theta$, giving us a planar joint geometry. In order to 


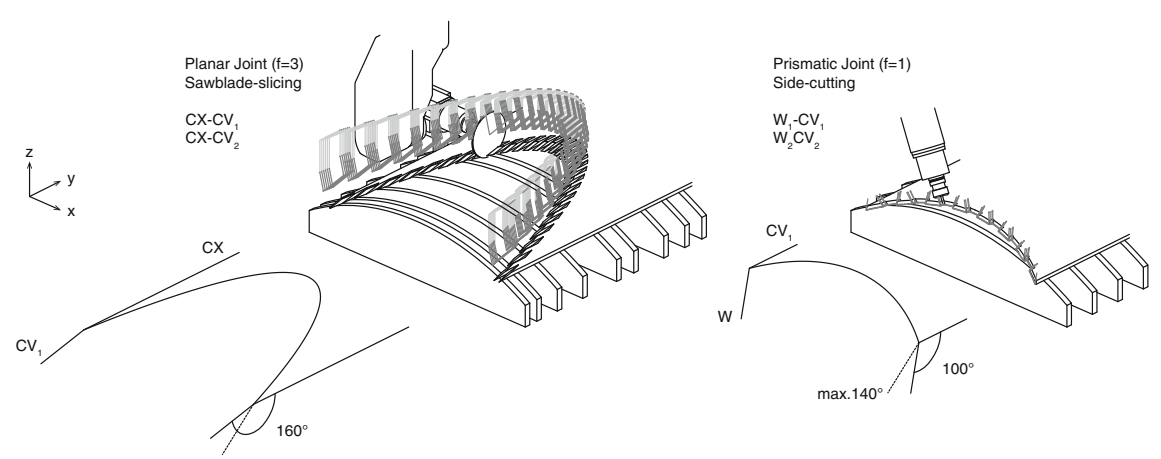

Fig. 5 Joint fabrication (tool path plot). Left planar "finger joint-type" connection (connections CV-CX), Right prismatic "dovetail-type" joint (connections W-CV)

cut the open slots of these joints, we used a saw blade-slicing technique instead (Fig. 5, left side), making multiple vertical cuts for each slot. This method allows us to fabricate very obtuse folds on simple, non-cantilevering supports.

\section{Preliminary Structural Analysis}

A preliminary structural analysis task has been performed in order to determine an initial value for the panel thickness and assess structural performance of the curved geometry to support relevant loads. Results from this primary engineering stage would let choose (or design) the appropriate laminate product as well as to ensure that enough degrees of freedom are linked in order to properly transmit structural effort between panels. A Finite Element shell model has been established from the mid-surface of panels, with local joints between curved panels simulated as rotational and translation links acting on local axis. For each joint the local axis $\mathbf{x}$ is the tangent to the joint 3D curve, the $\mathbf{y}$ axis is the bisector of two normal vectors of connecting mid-surfaces. The $\mathrm{z}$ axis is then defined by the right hand rule.

Panels at this preliminary structural design stage are considered as isotropic with Young Modulus $\mathrm{E}=7,800 \mathrm{MPa}$ (a conservative value near to long term modulus of timber) and Poisson ratio $v=0.3$. Structure is a temporary installation loaded for combination of self-weight and lateral as well as transversal wind load cases. Results for Ultimate Limit State and Service Limit State propose $77 \mathrm{~mm}$ thickness to be a safe initial size for the prototype (Fig. 6). This preliminary dimension will be confirmed through a detailed model once connection technology and the panel laminate product have been selected through the investigations which would be carried out on the 1:5 scale prototype. 


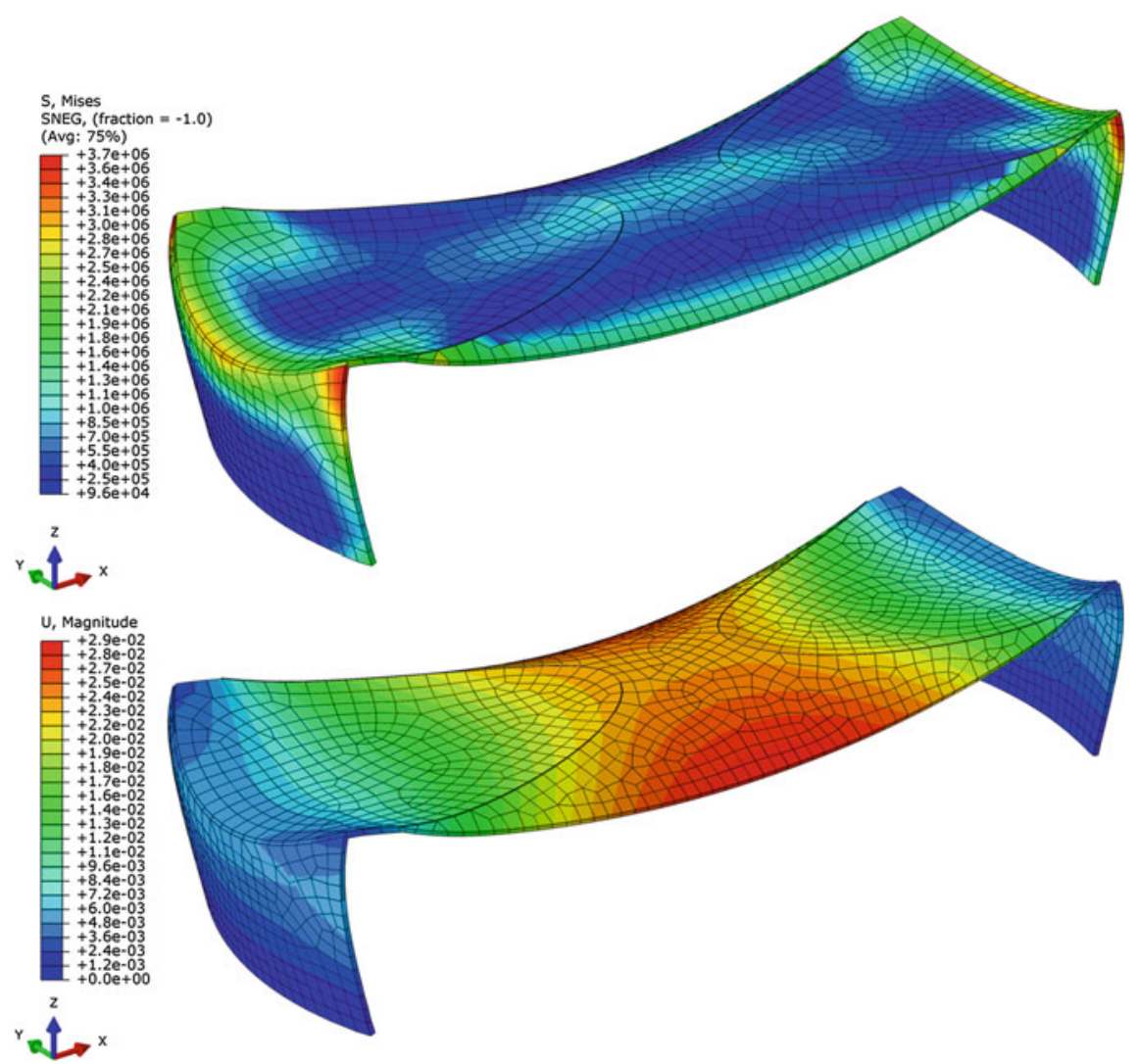

Fig. 6 Preliminary structural analysis results under the self-weight load case. Top Von-Mises stress distribution. Bottom total deformation

\section{Prototype at Scale 1:5}

The fabrication methods and the assembly of the parts were tested on a smaller prototype at scale 1:5 before applying it to the full-scale prototype. The curved CLT, which is not available at the scaled thickness, was replaced by a laminate of thin plywood panels. A MAKA mm7s 5-axis CNC router was used for the formatting of the panels and for the cutting of the joints.

Due to the curved geometry of the parts, custom-made supports (Fig. 7) were required for the curved panels, which served simultaneously as a mold for our curved laminate.

A convex support was built for the parts $\mathrm{CX}, \mathrm{W}_{1}$ and $\mathrm{W}_{2}$, and a concave support for the parts $\mathrm{CV}_{1}$ and $\mathrm{CV}_{2}$. An additional concave support was built for the saw blade-slicing technique, which requires access from above on the convex and the concave panel. The molds were fabricated from laminated veneer lumber panels 


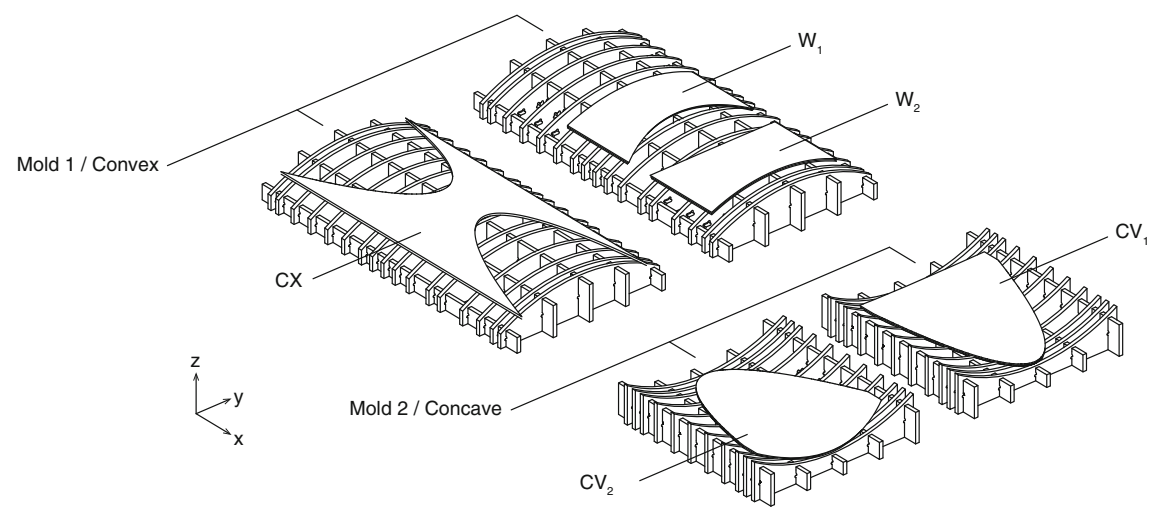

Fig. 7 Concave and convex mold for the lamination and formatting of the curved panels at scale 1:5
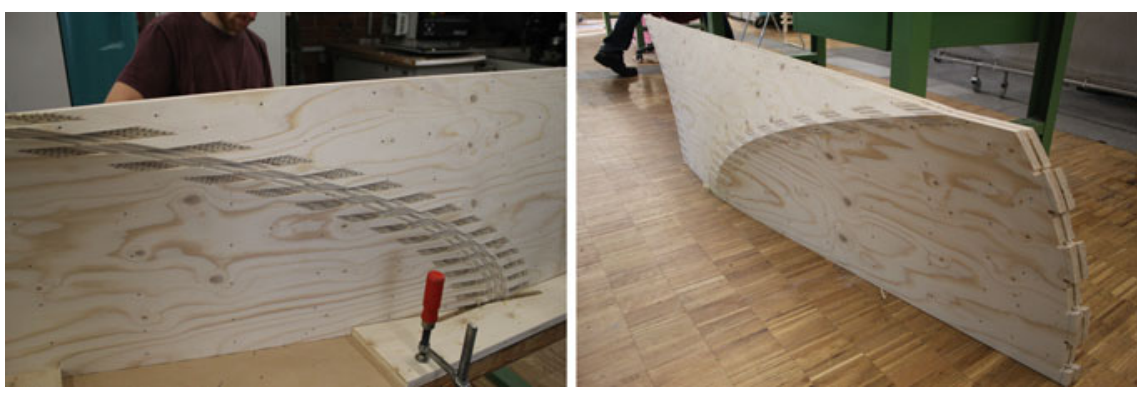

Fig. 8 Planar finger-type joints were used for the connections $\mathrm{CV}_{1}-\mathrm{CX}$ and $\mathrm{CV}_{2}-\mathrm{CX}$. These joints were fabricated with a saw blade-slicing technique on a 5-axis $\mathrm{CNC}$ router

(LVL) and assembled with half-lap joints. In order to provide a precise calibration of the supports, a previously added oversize was removed through spot facing along the ribs on the CNC-router.

Figure 8 shows the assembly of the parts $\mathrm{CX}$ and $\mathrm{CV}_{1}$ on the small-scale prototype: The short segments of the planar joint provide a large contact area and gluing surface between the panels.

\section{Full-Scale Prototype}

With the initial thickness determined at the preliminary engineering stage (see Sect. 5), a special laminate product has been designed by the manufacturer to fulfill the requirement regarding the thickness/radius ratio. The selected CLT panels are five layer symmetric laminates with total thickness of $77 \mathrm{~mm}$. 
(a)

(b)
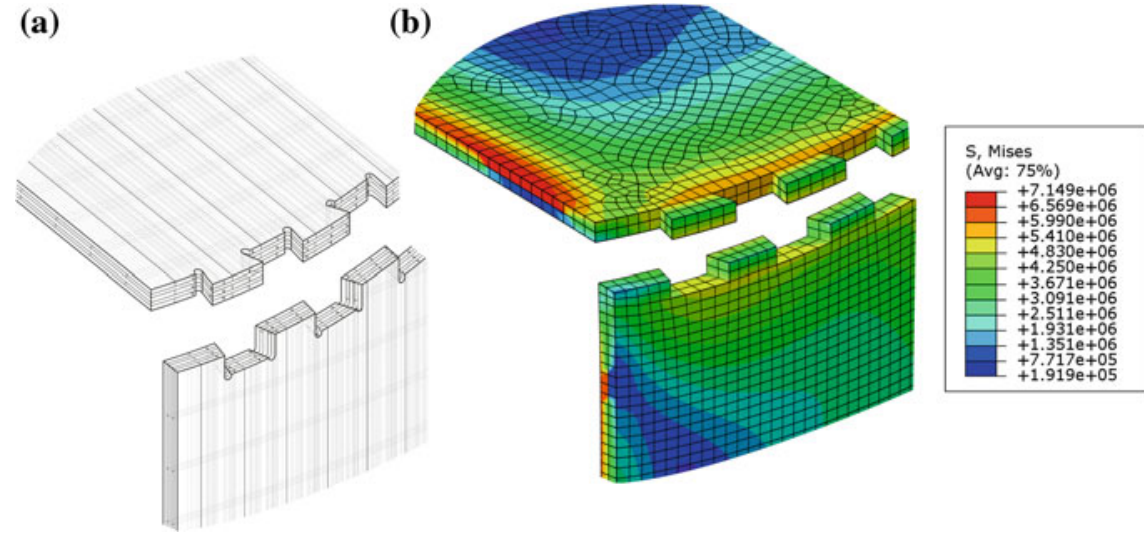

Fig. 9 Von-Mises stress distribution for Dovetail-type connections $\mathrm{CV}_{1}-\mathrm{W}_{1}$ and $\mathrm{CV}_{2}-\mathrm{W}_{2}$ on the full-scale prototype. Results are generated from a full three dimensional finite element simulation

As the next step, a full three-dimensional Finite Element model has been developed with this precise layer configuration and the mechanical properties provided by the manufacturer. The glued areas over the connectors have been considered as surface tie constraints. The goal of this detailed engineering step was to investigate local panel interaction over the connectors as well as to confirm the thickness of the CLT panel regarding the structural safety. The structural analysis confirmed the initial design thickness. The design issue was mainly to overcome deformation due to Service Limit State load combinations. The local and overall Von-Mises stress distribution is shown respectively in Figs. 9 and 10.

Having verified the structural parameters of the full scale prototype through the detailed analysis, the fabrication process was carried out at the facilities of the manufacturer. Figure 11 shows the fabrication of the full-scale prototype at the CLT panel production and processing facilities. The KUKA KR250 robot router with an additional linear-axis table allowed for the precise 5-axis fabrication of the joints at full scale. Its large workspace with a length of more than $30 \mathrm{~m}$ was essential for the realization of the prototype. The sides of the curved CLT panels were formatted with an $800 \mathrm{~mm}$ saw blade (Fig. 11, right side) and the dovetailtype joints $\mathrm{W}_{1}-\mathrm{CV}_{1}$ and $\mathrm{CV}_{2}-\mathrm{W}_{2}$ were cut with a $25 \mathrm{~mm}$ turn-blade knife shanktype cutter.

For the joints $\mathrm{CV}_{1}-\mathrm{CX}, \mathrm{CX}-\mathrm{CV}_{2}$, a different joinery technique was used for the full-scale prototype: 30 slots, each with a width of $30 \mathrm{~mm}$ were cut into the curved panels to be connected. Subsequently, connector elements with rounded edges (Fig. 12, right side), were fabricated from cross-laminated spruce LVL with a Hundegger K1 joinery machine. During the assembly of the full-scale prototype, these connectors were first used to align the roof segments, before gluing them in with a 1K-PUR adhesive. This alternative technique was chosen to reduce the machining time at full-scale. The robot router's higher sensitivity to vibrations required a lower feed rate compared to the $\mathrm{CNC}$ portal router (factor 0.2 ), while 


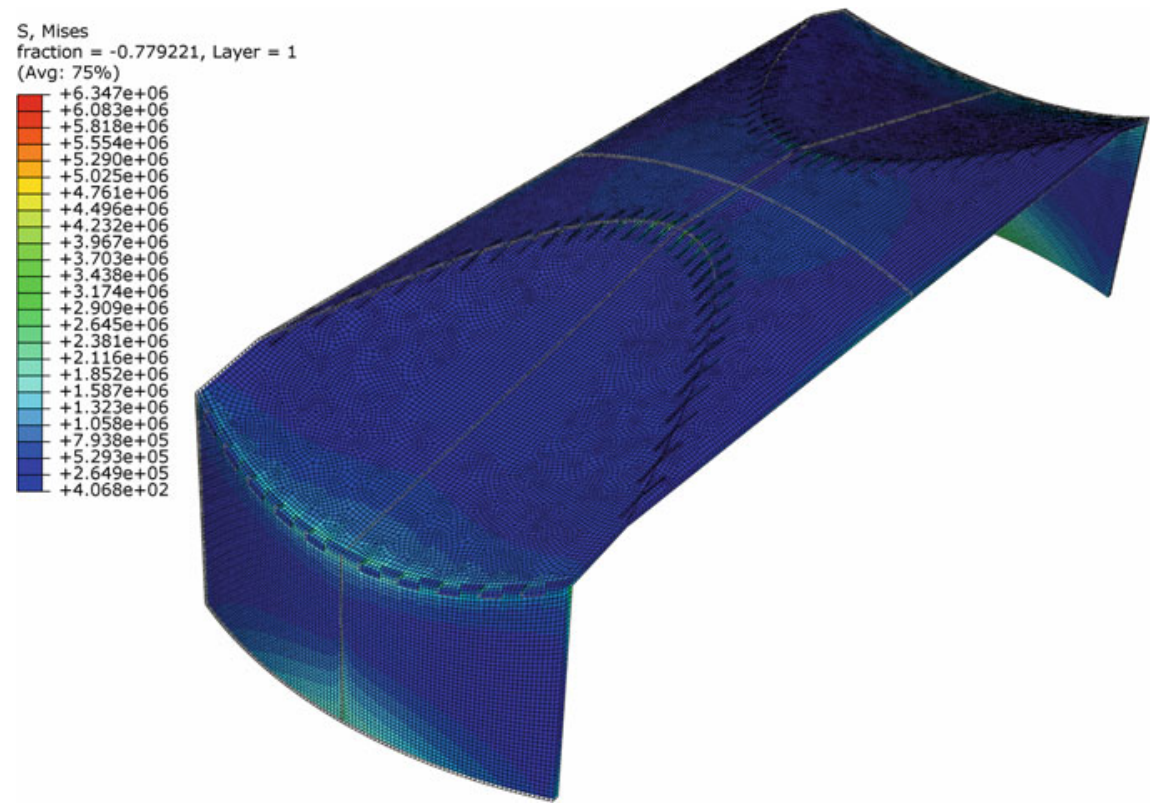

Fig. 10 Von-Mises stress distribution for detailed model
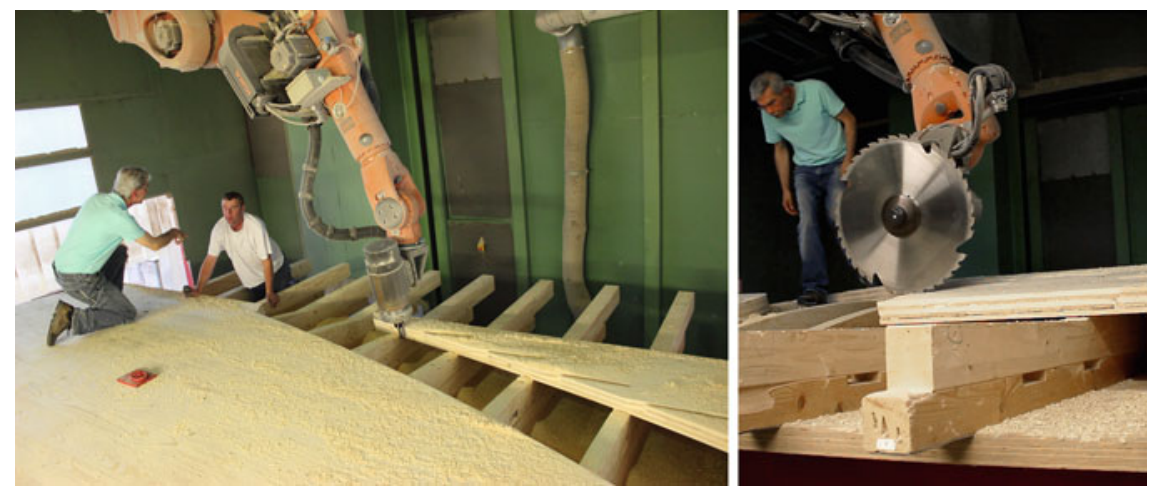

Fig. 11 Robot fabrication of the full-scale prototype. Left fabrication of the joint $\mathrm{CV}_{1}-\mathrm{CX}$ using a $25 \mathrm{~mm}$ shank-type cutter. Right formatting of the side of the panels using a saw blade with a diameter of $800 \mathrm{~mm}$

the length of the tool paths increased by the factor 5 compared to the prototype at scale 1:5.

The slots for the LVL connectors could be fabricated without access from above on the concave panels $\mathrm{CV}_{1}$ and $\mathrm{CV}_{2}$. This allowed for all formatting and joinery to be done on one convex support. An additional concave support was not necessary for the fabrication of the full-scale prototype. 



Fig. 12 Left assembly of the roof panels: shell prototype ready for the insertion of the LVL connectors (connections CV-CX). Right schematic drawing of the connector elements made from cross-laminated LVL panels. The slots have been cut with a $30 \mathrm{~mm}$ shank-type turn blade-knife cutter

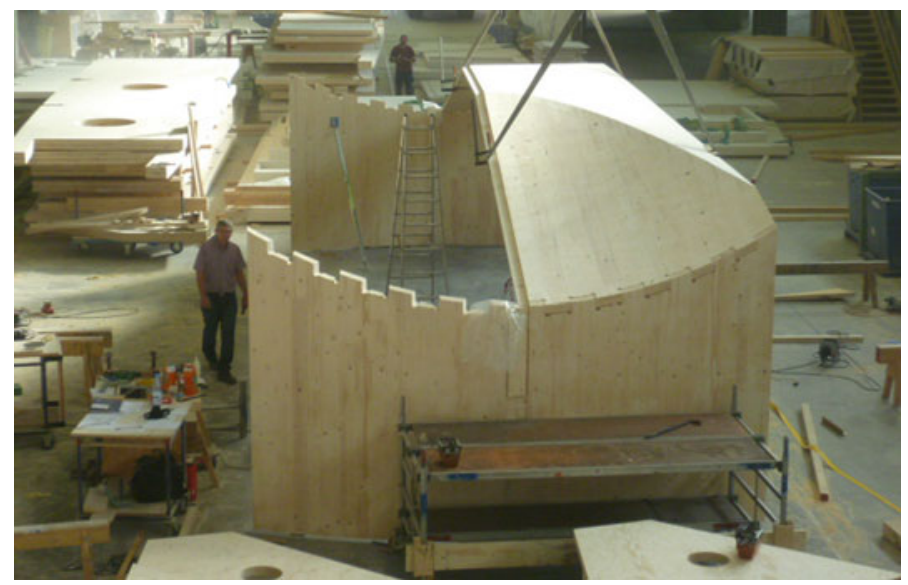

Fig. 13 Assembly of the dovetail-type connectors between the wall panels and the roof panels (Connections CV-CX)

After the panel formatting, the different parts of the pavilion were assembled. The three parts of the roof $\mathrm{CV}_{1}, \mathrm{CX}$ and $\mathrm{CV}_{2}$ were assembled in a first step and lifted with a portal crane. Afterwards, the vertical elements W1 and W2 were positioned and an adhesive was applied to the faces of the dovetail-type connections $\mathrm{W}_{1}-\mathrm{CV}$ and $\mathrm{CV}_{2}-\mathrm{W}_{2}$. Finally the complete roof was lowered into position from above with a portal crane (Fig. 13).

For weather protection, the entire shell has been treated with preservation for coniferous wood, before it was transported to its destination at the Academy of Architecture at the Universita della Svizzra Italiana (USI). After its final assembly 
on site, the structure was presented to the public in September 2013. To date, the temporary design is still exhibited on the campus of USI. Its exposure to the outdoor conditions is being documented.

\section{Conclusions/Towards an Integration of Joints}

Instead of using additional stiffening elements, the thin-shell presented in this chapter achieves its wide span with an integrated approach. The design utilizes the geometry of curved CLT panels and combines it with the technique of curvedfolding.

The approach of integrated performance has been continued on the design of the joints: The connections were fabricated during the formatting of the panels and performed simultaneously as guides for the positioning and alignment of the pieces. The precise fabrication and the mono-material approach allowed for a visible application of the joints. This was not only useful for the aesthetic appearance of the prototype, but also for the simple monitoring of the condition of the joints.

Three different techniques were used for the joinery, all of which take advantage of computational fabrication technology for the fabrication of the joints and utilize digital geometry processing tools for the generation of both the joint geometry and the machining code.

The side-cutting technique used for the fabrication of the dovetail-type $\mathrm{W}-\mathrm{CV}$ joints demonstrates an integrated jointing method for the connection of curved CLT panels. Compared to a butt joint or miter joint geometry, it offers advantages such as a precise and fast alignment and the machining time is only slightly increased. The technique allows for the jointing of fold angles within a range of $50^{\circ}$ and $130^{\circ}$.

The saw blade-slicing technique allows for the jointing of more obtuse fold angles, but it requires additional access from above on the concave side of one panel. Both a convex and a concave support are required. The number of saw blade cuts for the open slots of the joints results from the total edge length divided by the saw blade thickness. Fast routers with a high feed rate are beneficial for the fabrication of this type of joint.

The LVL connector technique demonstrates an alternative method for the jointing of curved-folds with obtuse fold angles $\varphi$. It allows for fast fabrication and all joints were cut on a single convex support, but the manual assembly of the LVL connectors is more time-consuming compared to the dovetail joints.

While each of the presented joinery techniques shows certain advantages and disadvantages, we found the side-cutting technique to be a particularly effective and universal method for the jointing of curved CLT panels with a robot router. In general, the robot-manufactured joints allowed for the realization of our prototype, but we believe the construction demonstrates only one of many possible applications for curved-folded CLT structures. 


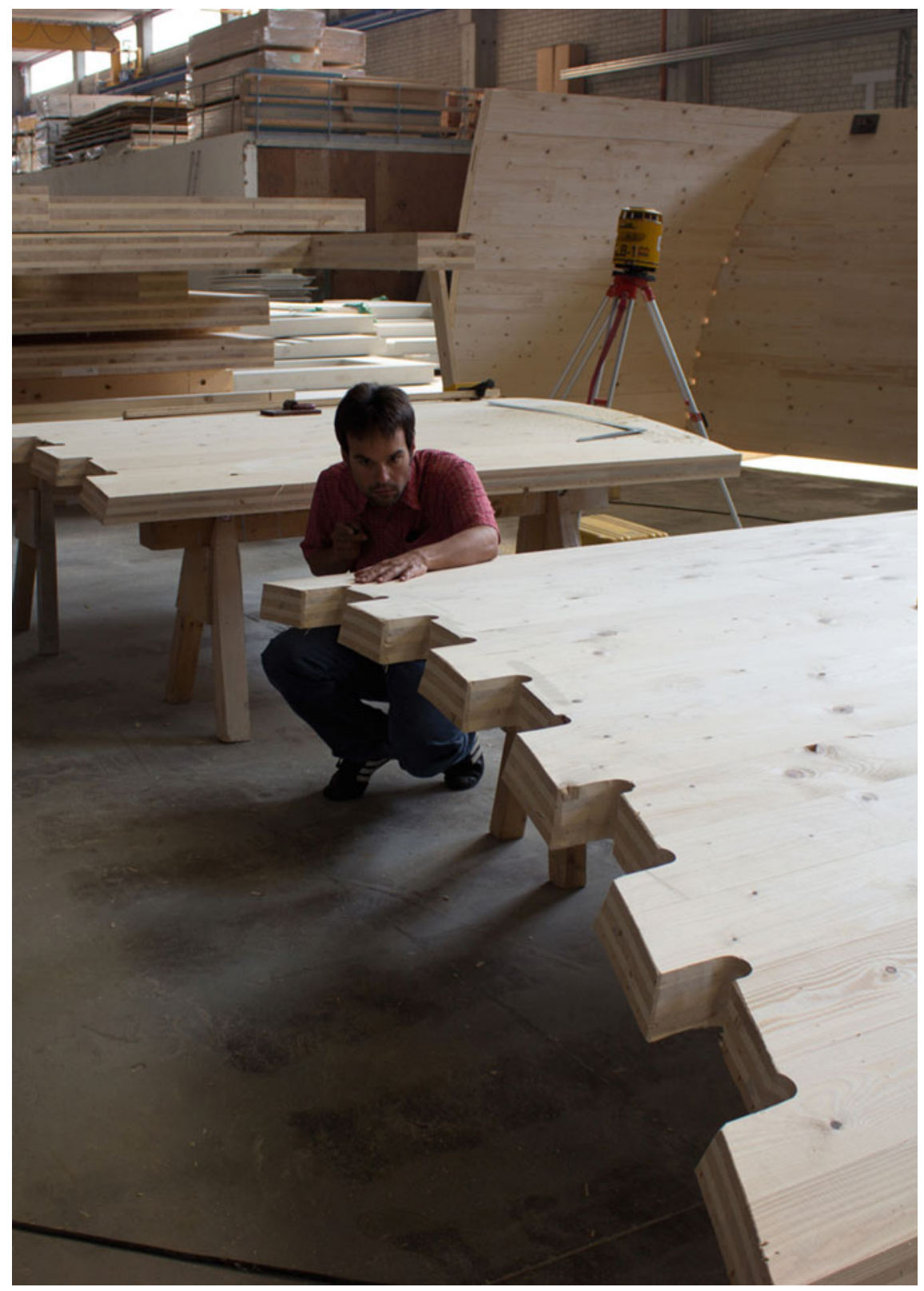




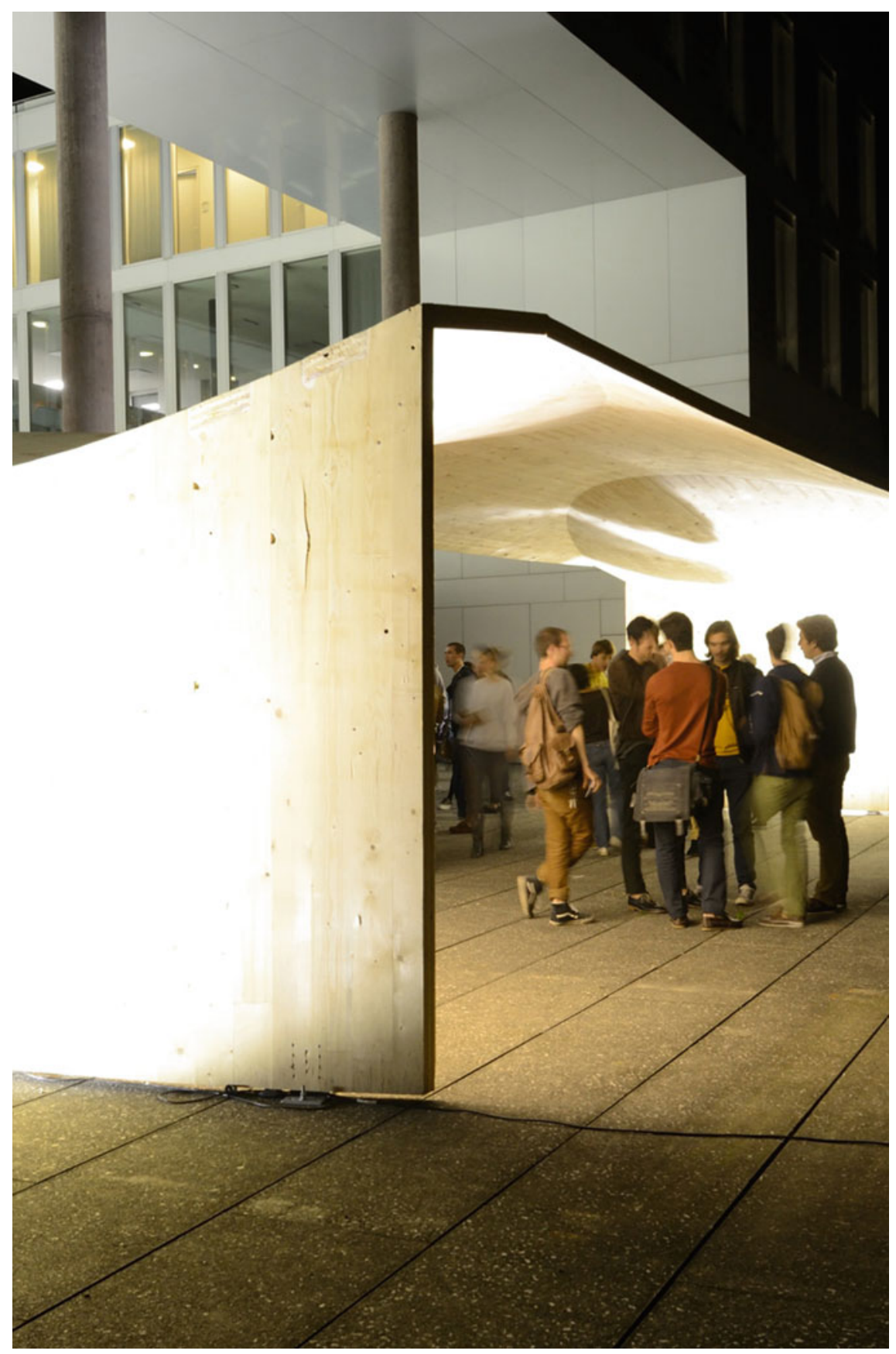




\section{References}

Buri H, Weinand Y (2010) Origami aus Brettsperrholz, DETAIL Zeitschrift für Architektur + Baudetail 2010(10):1066-1068

EN 1995-1-1:2004 (2004) Eurocode 5: design of timber structures-part 1-1: general—common rules and rules for buildings, $\mathrm{p} 42$

Kilian M et al (2008) Curved folding. ACM Trans Graph 27(3):1-9

Koch P (ed) (1964) Wood machining processes, collections: wood processing series. Ronald, New York

Magna R et al (2013) From nature to fabrication: biomimetic design principles for the production of complex spatial structures. Int J Space Struct 28(1):27-39

Simek M, Sebera V (2010) Traditional Furniture Joinery from the Point of view of Advanced Technologies. In: Proceedings of the international convention of society of wood science and technology and United Nations economic commission for Europe-Timber Committee, Geneva, Switzerland, 11-14 Oct 2010 